\title{
Gait efficiency using the C-Leg
}

\author{
Michael S. Orendurff, MS; ${ }^{*}$ Ava D. Segal, BAS; Glenn K. Klute, PhD; Martin L. McDowell, CPO; \\ Janice A. Pecoraro, RN; Joseph M. Czerniecki, MD \\ Motion Analysis Laboratory, Center of Excellence for Limb Loss Prevention and Prosthetic Engineering, Rehabilita- \\ tion Research and Development, Department of Veterans Affairs Puget Sound Health Care System, Seattle, WA
}

\begin{abstract}
Microprocessor-controlled prosthetic knees are claimed to improve gait efficiency in transfemoral (TF) amputees. This hypothesis was tested in a prospective randomized crossover trial that compared the Mauch SNS knee and the C-Leg microprocessor-controlled knee in eight TF amputees. The subjects were given a 3-month acclimation period in each knee. Then, their net oxygen cost $(\mathrm{mL} / \mathrm{kg} / \mathrm{m})$ was measured while they walked overground at four speeds in random order: $0.8 \mathrm{~m} / \mathrm{s}, 1.0 \mathrm{~m} / \mathrm{s}, 1.3 \mathrm{~m} / \mathrm{s}$, and self-selected walking speed (SSWS). The C-Leg caused small reductions in net oxygen cost that were not statistically significant compared with the Mauch SNS at any of the walking speeds $(p>0.190)$. Subjects chose higher SSWSs with the C-Leg compared with the Mauch SNS (mean \pm standard deviation = $1.31 \pm 0.12 \mathrm{~m} / \mathrm{s}$ vs $1.21 \pm 0.10 \mathrm{~m} / \mathrm{s}$, respectively, $p=0.046$ ) but did not incur higher oxygen costs $(p=0.270)$, which suggests greater efficiency only at their SSWS.
\end{abstract}

Key words: amputee, biomechanics, C-Leg knee, efficiency, gait, microprocessor control, oxygen cost, prosthetic knee, transfemoral, walking.

\section{INTRODUCTION}

Transfemoral (TF) amputees are less efficient ambulators and demonstrate a 27 to 88 percent increase in metabolic cost during walking compared with intact individuals [1-2]. This increased metabolic cost may be related to the mass asymmetry of the limbs or the absent sensorimotor control of the knee/ankle/foot mechanism, which leads to excessive cocontraction, abnormal trunk and limb movement, or abnormal energy transfer [1-2]. TF amputees use specific adaptive strategies in knee motion and lower-limb joint loading. Increased muscle activity at the intact limb and hips further stabilizes the knee during prosthetic stance and compensates for the lack of ankle-power generation by the prosthetic foot [3]. Because of these additional challenges with ambulation, TF amputees on average walk slower compared with intact individuals [2,4], perhaps to minimize ambulation costs or remain within normal energy cost limits [4].

Presumably, advancements in prosthetic technology are designed to lessen biomechanical asymmetries associated with amputation and maximize gait efficiency. The microprocessor-controlled prosthetic knee is a recent development that uses onboard electronic sensors to collect real-time data and control stance and swing phase movements. The manufacturers claim that this novel technology automatically adjusts to variable gait and terrain and alleviates many of the problems associated with TF amputation by minimizing compensation of the intact

\footnotetext{
Abbreviations: ANOVA $=$ analysis of variance, IP $=$ Intelligent Prosthesis, IRB = institutional review board, SD = standard deviation, SSWS = self-selected walking speed, TF = transfemoral, VA = Department of Veterans Affairs.

*Address all correspondence to Michael S. Orendurff, Motion Analysis Laboratory, Center of Excellence for Limb Loss Prevention and Prosthetic Engineering, Department of Veterans Affairs Puget Sound Health Care System, 1660 South Columbian Way, Seattle, WA 98108; 206-2776358; fax 206-764-2808. Email: morendurff@hotmail.com DOI: 10.1682/JRRD.2005.06.0095
} 
limb. In addition, results of subjective questionnaires have been generally positive, with reports of overall decreased fatigue [5]. However, little objective data have demonstrated increased gait efficiency for TF amputees using microprocessor-controlled knees.

A few previous studies have measured the energy expenditure during ambulation of amputees wearing a microprocessor knee called the Intelligent Prosthesis (IP) [6-8]. Buckley et al. [6] and Taylor et al. [7] were only able to suggest trends of decreased metabolic cost associated with the IP since they examined one and three subjects, respectively. Chin et al. tested more subjects $(n=8)$ and compared IP users with nondisabled subjects [8]. Although IP users still demonstrated increased energy expenditure compared with the nondisabled subjects, the 24 percent increase was less than previously documented for TF amputees. Datta et al. recently compared conventional and microprocessor-controlled knees in amputees at several walking speeds and found significantly reduced oxygen costs at slower walking speeds; however, they did not calculate net oxygen costs and none of the subjects reached steady state at any of the speeds [9]. Subtraction of the subjects' oxygen cost at rest from the oxygen cost at slower walking speeds might have eliminated the statistically significant difference at slower walking speeds observed by Datta et al. [9].

Metabolic rate is quite variable, especially across subjects. A within-subject design minimizes this variability and is better suited for detecting differences between prosthetic devices. Schmalz et al. performed a within-subject comparison of TF amputees who wore a conventional hydraulic single-axis knee joint (3Cl) and a microprocessor-controlled knee (C-Leg); they reported a 6 percent reduction in oxygen consumption rate at slower walking speeds ( 0.5 to $1.1 \mathrm{~m} / \mathrm{s})$ but no significant difference at faster walking speeds (1.0 to $1.4 \mathrm{~m} / \mathrm{s}$ ) [10]. Schmalz et al. did not compare oxygen cost (milliliter/kilogram/meter) between the conventional hydraulic and the microprocessor-controlled knees, only oxygen consumption rate (milliliter/kilogram/minute), and this protocol did not include a lengthy acclimation period during which the amputees might adapt their gaits to the microprocessor-controlled knee.

This study determined whether the C-Leg microprocessor-controlled prosthetic knee would improve gait efficiency in traumatic TF amputees more than the Mauch SNS knee following a long-term acclimation period.

\section{METHODS}

Eighteen traumatic TF amputees gave their informed consent to participate in this study. The protocol was approved by the University of Washington human subjects committee for the Department of Veterans Affairs (VA) Puget Sound Health Care System. Subjects from the general population of TF amputees in the metropolitan Seattle area were recruited by use of institutional review board (IRB)-approved flyers, word of mouth, and an IRB-approved registry of TF amputees at the VA Puget Sound Health Care System. The experiment involved the testing of each subject's gait efficiency with the Mauch SNS knee (Ossur, Reykjavik, Iceland) and the C-Leg microprocessor-controlled prosthetic knee (Otto Bock, Minneapolis, Minnesota) after 3 months of acclimation. An experienced Otto Bock-trained, C-Leg-certified prosthetist created identical sockets for subjects with a distal attachment that could accommodate either the C-Leg or the Mauch SNS knees; three subjects already had adequate sockets and did not require socket fabrication. In all cases, the same socket was used in both trials and only the knees were changed for the C-Leg and Mauch SNS trials. Socket fitting required that the subjects visit the prosthetist three to five times over a 2-week period. The specific damping and alignment characteristics of the CLeg and Mauch SNS knees were adjusted by the prosthetist with input from the subjects.

The subjects were evaluated after they had worn only the specific limb for all activities over 3 months; a coin-flip determined whether the subjects would start with the Mauch SNS or the C-Leg. This determination occurred after the subjects had had their experimental sockets fabricated and had achieved a comfortable fit that satisfied both the subject and the prosthetist. All subjects had been long-term ( $>3$ years) Mauch SNS users prior to the study. After the 3month acclimation period in the specific limb, the subjects' oxygen consumption rate at a range of walking speeds was measured with a VmaxST portable telemetered metabolic measuring system (Sensormedics, Yorba Linda, California). Subjects were also tested in an array of domains, including gait biomechanics, cognitive interference during ambulation, ratings of perceived exertion, 1-week step counts, fatigue and prosthetic preference questionnaires, and timed stair and ramp courses, but only the oxygen cost during walking is reported in this article. Results of the gait biomechanics [11] and cognitive interference [12] assessments are published elsewhere. The tests were conducted at the same 
time of day for each subject, and subjects ate the same meal prior to the oxygen consumption rate test. The VmaxST was calibrated according to the manufacturer's procedure prior to every test. The subjects walked overground at four speeds in random order: $0.8 \mathrm{~m} / \mathrm{s}, 1.0 \mathrm{~m} / \mathrm{s}, 1.3 \mathrm{~m} / \mathrm{s}$, and a previously chosen self-selected walking speed (SSWS). Each subject chose a SSWS by completing 10 trials on an $11 \mathrm{~m}$ straight walkway. Timing lights measured the subject's walking speed over $2 \mathrm{~m}$ at the center of the walkway, and the average of the 10 trials was recorded as the SSWS.

Each subject's walking speed was monitored with a velocity-recording cart (which was named "Velocart") that was pushed by an investigator alongside the subject during oxygen consumption measurement. A small electric generator (SB-740A-7; Servo-Tek, Hawthorne, New Jersey) was attached to one wheel of the Velocart and produced a voltage proportional to speed. The voltage was sampled at $1,000 \mathrm{~Hz}$ with a Personal Computer Memory Card International Association data acquisition card (National Instruments, Austin, Texas) attached to a laptop computer. Target speeds could be programmed into the interface (LabView, National Instruments, Austin, Texas) and were represented as wide red lines on an analog display; the width of the line represented \pm 5 percent of the speed. The instantaneous speed was displayed as an overlaid thin black line so that the investigator could ensure that the Velocart and subject maintained the desired speed. During testing at SSWS, the investigator used the Velocart to ensure that the subjects maintained their previously chosen SSWS. Subjects were instructed to remain within arm's length of the Velocart during walking trials in a 3.2-m wide hallway that formed a 60 m rectangular course with four corners each lap. Subjects followed a wide arc around the corners to minimize drops in walking speed at the corners. A speed record of each trial was stored. Breath-by-breath oxygen consumption rate was telemetered to a stationary laptop computer and monitored in real time throughout the trial. Subjects began the trial seated until they reached steady state. Then the subjects began walking at the first selected speed paralleled by an investigator pushing the Velocart. Subjects continued walking at the target speed until a minimum 2 minutes of steady state oxygen consumption rate was observed, which usually occurred within 10 minutes of the start of the trial. Steady state oxygen consumption was ensured with $t$-tests that compared the four 30-second intervals of the final 2 minutes with one another (for a total of six $t$-tests). Subjects returned to the seated position until they again achieved their baseline oxygen consumption rate and then moved to the next speed. This process was repeated for all four walking speeds.

Gross oxygen consumption rate was normalized to body weight (milliliter $\mathrm{O}_{2} / \mathrm{kilogram} /$ minute) for each trial and converted to net oxygen consumption rate by subtraction of each subject's average resting oxygen consumption rate for all walking trial measurements. Net oxygen consumption data was converted into oxygen cost (milliliter $\mathrm{O}_{2} /$ kilogram/meter) by dividing net oxygen consumption by speed (meter/minute). A two-way, repeated measures analysis of variance (ANOVA) (4 speeds $\times 2$ knees) was used for determining whether subjects wearing the C-Leg and Mauch SNS knees differed in net oxygen cost at walking speeds of $0.8 \mathrm{~m} / \mathrm{s}, 1.0 \mathrm{~m} / \mathrm{s}$, $1.3 \mathrm{~m} / \mathrm{s}$, and SSWS. Scheffe's post hoc tests were used for specific comparisons. SSWS was also compared between the C-Leg and Mauch knees with a one-way ANOVA.

\section{RESULTS}

Of the original 18 subjects, only 8 completed the full protocol. Four subjects withdrew prior to socket fitting and cited the time commitment of the study as too demanding. Two subjects could not be fit for their CLegs: one through-knee amputee with short stature and a long residual limb (the pylon that houses the strain gauges and informs the microprocessor could not be cut short enough without destroying the sensors) and one with a short residual limb and persistent socket-limb transverse-plane rotation problems during gait. One subject withdrew from the study while he recovered from surgical resection of a neuroma on his residual limb. One subject was dropped from the study because he had additional neurological involvement that was not identified until his first gait analysis. One subject achieved good socket fit but could not acclimate to the C-Leg and felt so unstable that he withdrew. Finally, one subject completed the first half of the study (Mauch SNS) but developed cancer and withdrew prior to C-Leg evaluation to successfully complete chemotherapy.

The remaining eight subjects (mean \pm standard deviation [SD] = age $48.5 \pm 10.2 \mathrm{yr}$, height $172.5 \pm 4.2 \mathrm{~cm}$, weight $80.1 \pm 10.5 \mathrm{~kg}$; one female; seven employed) who completed the study did not show any statistically significant differences in oxygen efficiency when they walked 
with the C-Leg compared with the Mauch SNS (Figure 1). The ANOVA revealed a main effect for knee type $(p=$ 0.270 ) that showed that the overall effect of the C-Leg on gait efficiency was not significant across all walking speeds. The C-Leg showed consistently lower mean oxygen costs at each speed, but the reductions were not substantial enough to achieve significance at any of the individual walking speeds compared with the Mauch SNS (Table 1). SSWS with the C-Leg was significantly faster than with the Mauch SNS (mean $\pm \mathrm{SD}=1.31 \pm 0.12 \mathrm{~m} / \mathrm{s}$ vs $1.22 \pm 0.10 \mathrm{~m} / \mathrm{s}$, respectively, $p=0.046$ ), but the oxygen cost did not significantly increase during ambulation at the faster walking speed ( $p=0.185$ ).

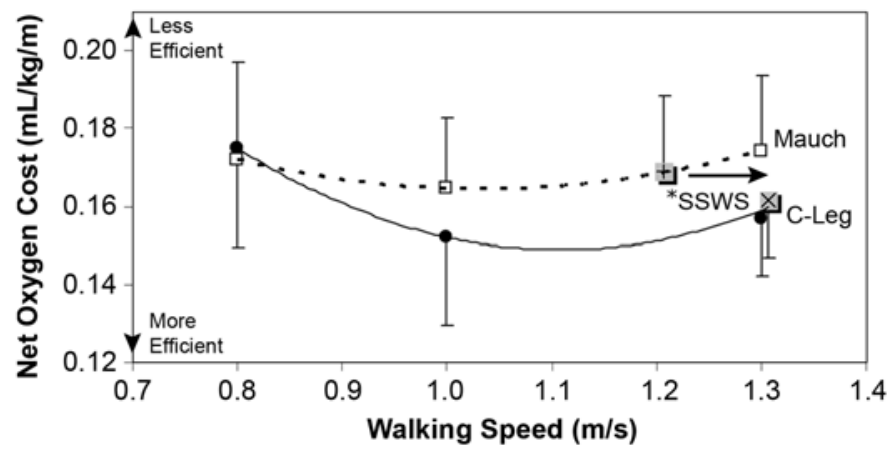

Figure 1.

Subjects' mean \pm standard deviation oxygen cost for Mauch SNS knee and C-Leg microprocessor-controlled knee over a range of walking speeds. No significant differences were detected in oxygen cost between two knees across all walking speeds. Equation for Mauch SNS (dotted line) was $y=0.1403 x^{2}-0.2899 x+0.3142\left(R^{2}=\right.$ 0.9977). Equation for C-Leg (solid line) was $y=0.2764 x^{2}-0.6124 x+$ $0.488\left(R^{2}=0.9731\right)$. ${ }^{*}$ Self-selected walking speed (SSWS) (shaded boxes) was significantly faster $(p=0.046)$ in the C-Leg than Mauch SNS without any significant increase in oxygen cost.
Individual results show that subjects $1,2,3$, and 4 were more efficient with the C-Leg; subjects 5, 6, and 7 had no change; and subject 8 was less efficient with the C-Leg (Figure 2). All but one of the eight subjects preferred the C-Leg and kept it as their primary prosthetic limb. Subject 6 chose to keep the Mauch SNS as his primary prosthetic limb. The prosthetic components used by each subject in both experimental conditions are shown in Table 2.

\section{DISCUSSION}

The study results were unexpected and show that, for this small cohort, the C-Leg did not improve gait efficiency, as reflected in the nonsignificant changes in oxygen costs over a range of walking speeds. This result is somewhat surprising since the adaptability of the C-Leg to any gait speed is often emphasized. Whatever alterations occurred in the motions or forces of the C-Leg, the residual limb, or the intact limb, these changes were not substantial enough to reduce oxygen cost across the range of speeds in this study. This result suggests that the gait of Mauch SNS users is as efficient as the gait of C-Leg users at approximately one-sixth of the dollar cost.

Some individuals, however, did show substantial reductions in oxygen cost across all speeds with the C-Leg, and this suggests that each individual may respond uniquely to a specific prosthetic prescription. Assessment of gait efficiency on an individual basis may provide the evidence necessary for provision of the optimal prosthetic prescription rather than one "best" device for all TF amputees.

The gross oxygen cost data were similar to previous work $[2,6,8,10]$, although some of the previous studies included very slow walking speeds (Figure 3). Comparing data from previous literature, the differences in gait

Table 1.

Paired comparisons of net oxygen cost (mean \pm standard deviation [SD]) for eight transfemoral amputees walking overground with C-Leg knee and Mauch SNS knee at four speeds. Also shown are participants' mean \pm SD self-selected walking speeds in each knee.

\begin{tabular}{lcccc}
\hline \multirow{2}{*}{ Speed (m/s) } & \multicolumn{2}{c}{ Net Oxygen Cost (mL/kg/m) } & \multirow{2}{*}{ p-Value } & \multirow{2}{*}{ Statistical Power } \\
\cline { 2 - 3 } & C-Leg & Mauch SNS & & \\
\hline 0.8 & $0.254 \pm 0.019$ & $0.235 \pm 0.022$ & 0.555 & 0.405 \\
1.0 & $0.214 \pm 0.020$ & $0.224 \pm 0.025$ & 0.246 & 0.127 \\
1.3 & $0.209 \pm 0.021$ & $0.225 \pm 0.027$ & 0.099 & 0.232 \\
SSWS & $0.210 \pm 0.016$ & $0.220 \pm 0.019$ & 0.185 & 0.184 \\
\hline Mean SSWS & $1.31 \pm 0.12$ & $1.21 \pm 0.10$ & $0.046^{*}$ & 0.391 \\
\hline *Self-selected walking speed (SSWS) was significantly faster with C-Leg without any increase in oxygen cost. & & \\
\hline
\end{tabular}


(a)
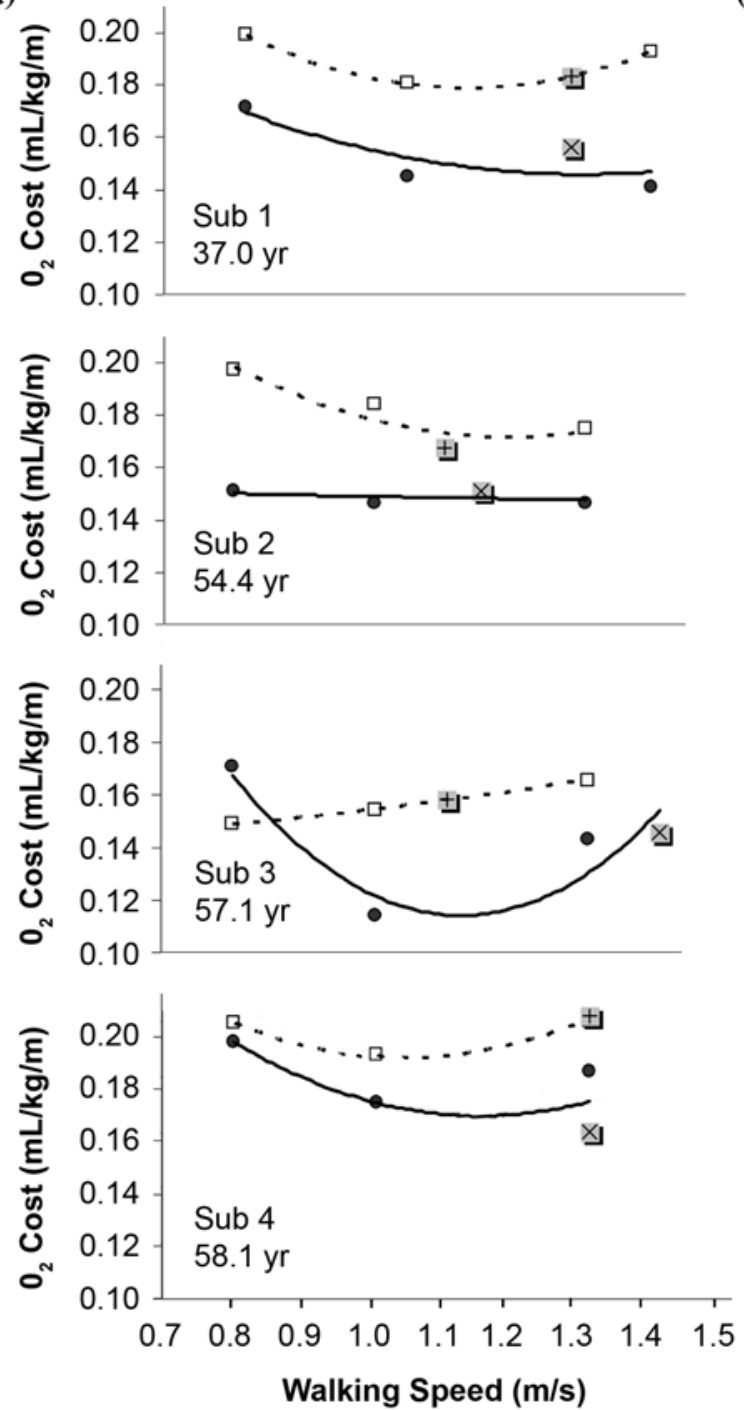

(b)
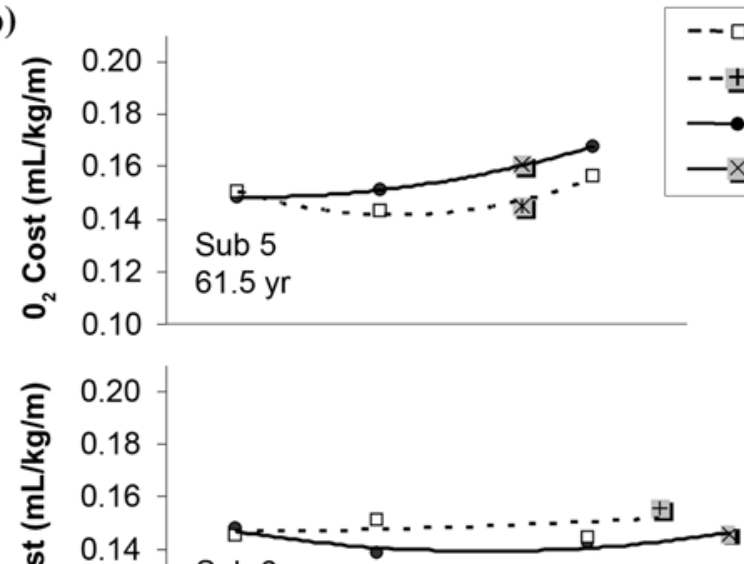

Sub 6

$\circ^{\mathrm{N}} 0.12-29.9 \mathrm{yr}$

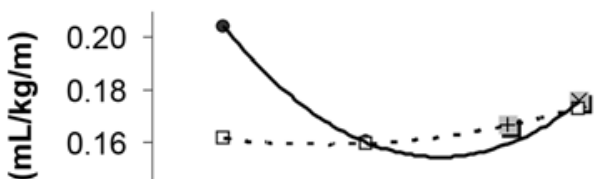

Sub 7

$59.2 \mathrm{yr}$

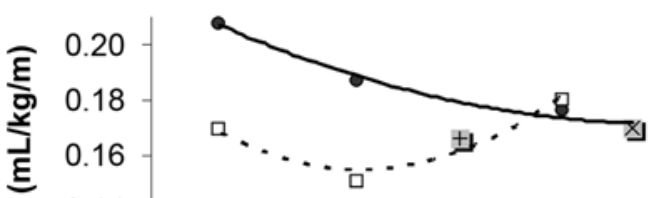

Sub 8

$32.2 \mathrm{yr}$

0.10

$\begin{array}{lllllllll}0.7 & 0.8 & 0.9 & 1.0 & 1.1 & 1.2 & 1.3 & 1.4 & 1.5\end{array}$

Walking Speed $(\mathrm{m} / \mathrm{s})$

Figure 2.

Individual subject's (Sub) net oxygen cost (walking minus resting) across a range of walking speeds with a C-Leg microprocessor-controlled knee and a Mauch SNS knee. (a) Subjects 1-4 showed improved gait efficiency with C-Leg. (b) Subjects 5-8 did not show improved gait efficiency with C-Leg. SSWS = self-selected walking speed.

Table 2.

Specific prosthetic components used by participating transfemoral amputees. Socket and suspension systems did not vary throughout study. However, the foot often varied to accommodate specific Otto Bock (OB) foot requirements for the C-Leg.

\begin{tabular}{clllll}
\hline Subject & Socket & Suspension & Foot Used with C-Leg & Foot Used with Mauch SNS & Efficiency with C-Leg* \\
\hline 1 & Carbon fiber & Suction & OB 1D25 Dynamic Plus & Seattle Lite Foot & $\Uparrow$ \\
2 & Carbon fiber & Pin & OB 1E40 LuXon Max & Flex-Walk Foot & $\Uparrow$ \\
3 & Thermo-plastic & Pin & OB 1D25 Dynamic Plus & Seattle Lite Foot & $\Uparrow$ \\
4 & Thermo-plastic & Pin & OB 1D25 Dynamic Plus & Seattle Lite Foot & $\Uparrow$ \\
5 & Thermo-plastic & Pin & OB 1D25 Dynamic Plus & Seattle Lite Foot & $\Leftrightarrow$ \\
6 & Carbon fiber & Suction & OB 1E40 LuXon Max & Flex-Walk Foot & $\Leftrightarrow$ \\
7 & Thermo-plastic & Pin & OB 1D25 Dynamic Plus & Seattle Lite Foot & $\Leftrightarrow$ \\
8 & Carbon fiber & Pin & OB 1C40 C-Walk & Flex-Walk Foot & $\Downarrow$
\end{tabular}




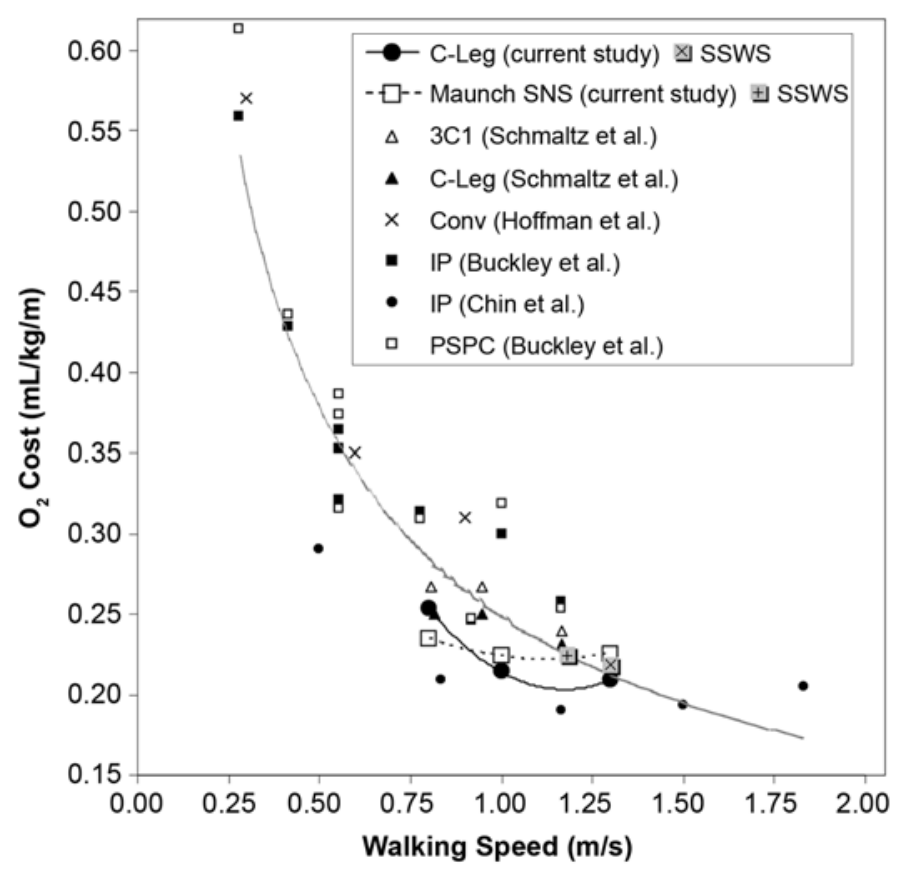

Figure 3.

Comparison of data from literature. Gross oxygen cost across range of walking speeds in transfemoral amputees using various prosthetic limbs. (Values converted to oxygen cost from oxygen consumption rate in some cases.) Sources: Schmalz T, Blumentritt S, Jarasch R. Energy expenditure and biomechanical characteristics of lower limb amputee gait: the influence of prosthetic alignment and different prosthetic components. Gait Posture. 2002;16(3):255-63 [PMID: 12443950]. Hoffman MD, Sheldahl LM, Buley KJ, Sandford PR. Physiological comparison of walking among bilateral above-knee amputee and able-bodied subjects, and a model to account for the differences in metabolic cost. Arch Phys Med Rehabil. 1997;78(4):385-92 [PMID: 9111458]. Buckley JG, Spence WD, Solomonidis SE. Energy cost of walking: comparison of "intelligent prosthesis" with conventional mechanism. Arch Phys Med Rehabil. 1997;78(3):330-33 [PMID: 9084360]. Chin T, Sawamura S, Shiba R, Oyabu H, Nagakura Y, Takase I, Machida K, Nakagawa A. Effect of an Intelligent Prosthesis (IP) on the walking ability of young transfemoral amputees: comparison of IP users with able-bodied people. Am J Phys Med Rehabil. 2003;82(6):447-51 [PMID: 12820787].

efficiency between microprocessor-controlled and conventional prostheses are not obvious. However, most authors did not calculate net oxygen cost by subtracting resting oxygen rates, which changes the relationships considerably. Previous work in which statistically significant improvements in gross oxygen consumption rate were observed with the C-Leg knee compared with the Otto Bock 3C1 conventional hydraulic knee did not include lengthy acclimation periods, and the C-Leg knee was always tested after the 3C1 knee [10]. That a short acclimation period would yield significant improvements with the C-Leg but a long acclimation period would not seems counterintuitive. Could it be that when first trying the C-Leg, subjects walk with their previous habitual kinematic and kinetic gait patterns and yield substantially improved gait efficiency but after a lengthy adjustment period in the C-Leg, kinematic and kinetic "laziness" sets in for some subjects and their gaits become less efficient? This hypothesis seems very unlikely but is possible. The nonrandom order of testing may also have played a role in the results of this previous work [10].

The socket and suspension system used for each subject in the Mauch SNS and C-Leg was identical, but the foot used was not (Table 2). Subjects had either a Seattle Lite Foot or a Flex-Walk Foot on their Mauch SNS. However, these feet were not approved for use on the C-Leg and were substituted with an Otto Bock-approved foot that the prosthetist judged most similar to the foot used on the Mauch SNS. In this way, this study had high clinical relevance, since the Mauch SNS was set to the "optimal configuration" conceived by the amputee, healthcare provider, and prosthetist, but sacrificed pure scientific objectivity by not controlling foot type.

The uncontrolled factor that likely had the greatest effect on walking efficiency with the C-Leg was programming of the mechanical characteristics of the knee. The C-Leg is programmed with Otto Bock Slider software. This process is performed by an authorized prosthetist who has completed an Otto Bock C-Leg training course. Programming is often done with considerable input from the amputee. The parameters include an initial zero load setting, maximum toe load, stance-phase damping, yielding extension damping, initial swing flexion damping, knee angle threshold, dynamic factor, and swing-phase extension damping (Figure 4). These parameters allow ". . . the prosthetist to control how dynamic the swing phase movements are, thus creating an optimal, energy-efficient gait for each amputee.”* Rigorous evaluation and a sound theoretical basis are likely needed before adjustment of the parameters on an individual's C-Leg for production of an optimal energy-efficient gait. Some of the amputees in this study gave feedback that would essentially have made the C-Leg a Mauch SNS, and some preferred that much more dynamic, responsive characteristics be programmed into

\footnotetext{
*Otto Bock, The Electronic 3C100/3C105 C-Leg Knee Joint System Instructions for Use, http://www.ottobockus.com
} 


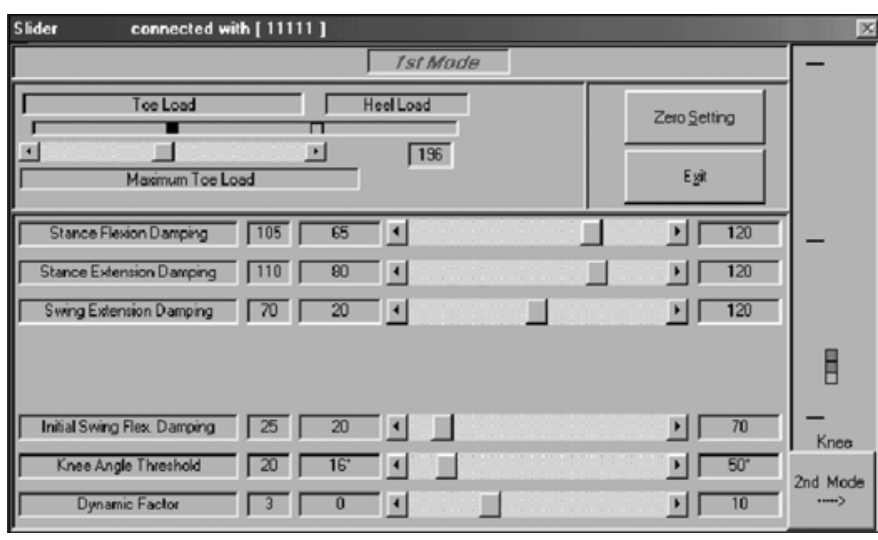

Figure 4.

Otto Bock Slider software dialog box for programming mechanical characteristics of C-Leg knee.

the C-Leg. If the knee is programmable for a wide array of characteristics, perhaps a particular set of characteristics results in more efficient gait as opposed to more stable gait and these were the characteristics that some amputees optimized during the programming sessions. This difference in programmed characteristics was not controlled for and may have had a large effect that was important only in hindsight. This aspect of the C-Leg has not been addressed in any research to date.

In Perry et al.'s case study of a bilateral TF amputee wearing the C-Leg and Mauch SNS knees [13], the subject showed a reduction from 304 percent of normal oxygen cost in the Mauch SNS to just 120 percent in the CLeg knee but had a persistently high $R$ value (1.03 vs 1.07); this indicated that the subject was ambulating above his or her anaerobic threshold with both knee types. Some subjects in previous studies walked at very slow speeds with very poor efficiency (Figure 3) [2,6]. The SSWSs chosen by the amputees in this study were consistently above their most efficient speed in both the Mauch and C-Leg limbs (Figure 2). These data suggest that gait efficiency may not always be the optimization goal for amputees.

Subjects gave the unsolicited opinion that they preferred the C-Leg primarily for its stumble recovery and secondarily for its capability to adapt to any walking speed they chose. The slightly increased walking speed on the C-Leg may be because of increased confidence rather than improved efficiency at the new, slightly faster walking speed. However, this new, faster walking speed, possibly the result of increased confidence with the C-Leg, resulted in lower oxygen cost compared with the Mauch SNS, though the reduction was not statistically significant.
Falls and fear of falling concern about one-half of amputees, and TF amputees are nearly three times more likely to fall as transtibial amputees [14]. Subjects may have favored the C-Leg over the Mauch SNS because of the C-Leg's perceived stability even though it did not improve gait efficiency. Previous authors have described this decision by TF amputees to favor stability over efficiency [15]. The capability of the C-Leg to resist knee flexion when the limb is suddenly loaded after catching the shoe on the ground appears to make recovery more likely in the event of a stumble. All the amputees in this study mentioned, without any prompting by the investigators, that stumble recovery was their favorite feature of the C-Leg.

\section{CONCLUSION}

The data from this study showed that, for some individuals, the metabolic cost of walking can be substantially reduced across a range of walking speeds with a C-Leg knee but not all individuals adapt equally well to the knee. These data suggest that a more objective, valid, and comprehensive assessment of each individual's gait is necessary before an optimal prosthetic limb can be prescribed, built, and programmed to improve TF amputees' walking efficiency.

\section{ACKNOWLEDGMENTS}

This material was based on work supported by the VA, Rehabilitation Research and Development Service, grants A2770I and A2661C.

The authors have declared that no competing interests exist.

\section{REFERENCES}

1. Gitter A, Czerniecki JM, Weaver K. A reassessment of center-of-mass dynamics as a determinate of the metabolic inefficiency of above-knee amputee ambulation. Am J Phys Med Rehabil. 1995;74(5):332-38. [PMID: 7576408]

2. Hoffman MD, Sheldahl LM, Buley KJ, Sandford PR. Physiological comparison of walking among bilateral aboveknee amputee and able-bodied subjects, and a model to account for the differences in metabolic cost. Arch Phys Med Rehabil. 1997;78(4):385-92. [PMID: 9111458] 
3. Czerniecki JM. Rehabilitation in limb deficiency. 1. Gait and motion analysis. Arch Phys Med Rehabil. 1996;77(3 Suppl):S3-8. [PMID: 8599543]

4. Waters RL, Perry J, Antonelli D, Hislop H. Energy cost of walking of amputees: the influence of level of amputation. J Bone Joint Surg Am. 1976;58(1):42-46. [PMID: 1249111]

5. Datta D, Howitt J. Conventional versus microchip controlled pneumatic swing phase control for trans-femoral amputees: user's verdict. Prosthet Orthot Int. 1998;22(2): 129-35. [PMID: 9747997]

6. Buckley JG, Spence WD, Solomonidis SE. Energy cost of walking: comparison of "intelligent prosthesis" with conventional mechanism. Arch Phys Med Rehabil. 1997;78(3): 330-33. [PMID: 9084360]

7. Taylor MB, Clark E, Offord EA, Baxter C. A comparison of energy expenditure by a high level trans-femoral amputee using the Intelligent Prosthesis and conventionally damped prosthetic limbs. Prosthet Orthot Int. 1996;20(2): 116-21. [PMID: 8876005]

8. Chin T, Sawamura S, Shiba R, Oyabu H, Nagakura Y, Takase I, Machida K, Nakagawa A. Effect of an Intelligent Prosthesis (IP) on the walking ability of young transfemoral amputees: comparison of IP users with able-bodied people. Am J Phys Med Rehabil. 2003;82(6):447-51. [PMID: 12820787]

9. Datta D, Heller B, Howitt J. A comparative evaluation of oxygen consumption and gait pattern in amputees using Intelligent Prostheses and conventionally damped knee swing-phase control. Clin Rehabil. 2005;19(4):398-403. [PMID: 15929508]
10. Schmalz T, Blumentritt S, Jarasch R. Energy expenditure and biomechanical characteristics of lower limb amputee gait: the influence of prosthetic alignment and different prosthetic components. Gait Posture. 2002;16(3):255-63. [PMID: 12443950]

11. Segal AD, Orendurff MS, Klute GK, McDowell ML, Williams RM, Turner AP, Pecoraro JA, Shofer J, Czerniecki JM. The gait mechanics of transfemoral amputees using the C-Leg compared to the Mauch SNS. J Rehabil Res Dev. In press 2006.

12. Williams RM, Turner AP, Orendurff MS, Segal AD, Klute, GK, Pecoraro JA, Czerniecki JM. Does having a computerized prosthetic knee influence cognitive performance during amputee walking? Arch Phys Med Rehabil. In press 2006.

13. Perry J, Burnfield JM, Newsam CJ, Conley P. Energy expenditure and gait characteristics of a bilateral amputee walking with C-leg prostheses compared with stubby and conventional articulating prostheses. Arch Phys Med Rehabil. 2004;85(10):1711-17. [PMID: 15468036]

14. Miller WC, Speechley M, Deathe B. The prevalence and risk factors of falling and fear of falling among lower extremity amputees. Arch Phys Med Rehabil. 2001;82(8): 1031-37. [PMID: 11494181]

15. Devlin M, Sinclair LB, Colman D, Parsons J, Nizio H, Campbell JE. Patient preference and gait efficiency in a geriatric population with transfemoral amputation using a free-swinging versus a locked prosthetic knee joint. Arch Phys Med Rehabil. 2002;83(2):246-49. [PMID: 11833030]

Submitted for publication June 8, 2005. Accepted in revised form September 30, 2005. 